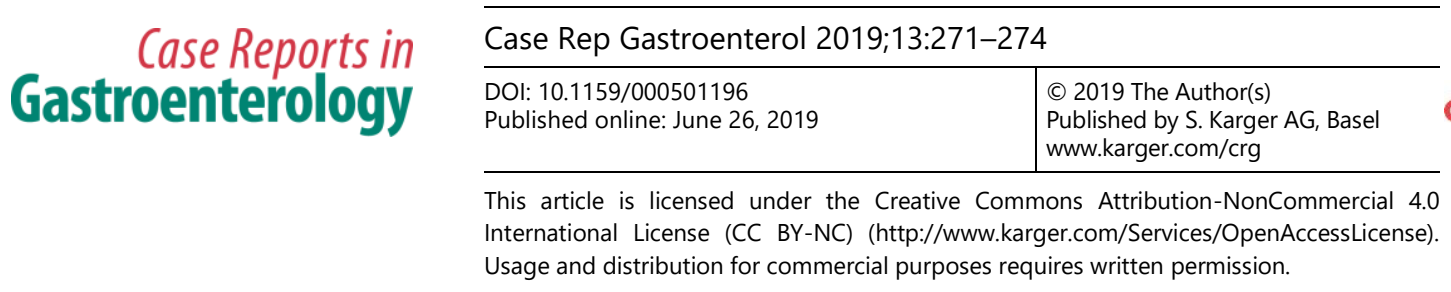

\title{
Melanocytic Nevus of the Colon
}

\author{
Noriyuki Uesugi ${ }^{a}$ Hiroo Tateno ${ }^{b}$ Hiroshi Yokoyama ${ }^{c}$ Shu Abe ${ }^{c}$ \\ Ryo Sugimoto ${ }^{\mathrm{a}}$ Tamotsu Sugai $^{\mathrm{a}}$ \\ aDepartment of Diagnostic Molecular Pathology, School of Medicine, Iwate Medical \\ University, Morioka, Japan; 'biagnostic Pathology Japan, Inc., Sendai, Japan; \\ 'Department of Gastroenterology, JR Sendai Hospital, Sendai, Japan
}

\section{Keywords}

Colon · Melanocytic nevus $\cdot$ Neural crest $\cdot$ Pathogenesis

\begin{abstract}
Here, we report a very rare case of melanocytic nevus of the colon. A 54-year-old woman with an unremarkable medical history visited our hospital for screening colonoscopy. Colonoscopy revealed a pigmented flat lesion in the ascending colon. Histological evaluation of a biopsy specimen revealed proliferation of pigmented cells in the lamina muscularis propria. On immunohistochemical analysis, pigmented cells were positive for S-100 protein and Melan-A expression, and a diagnosis of colonic melanocytic nevus was made. Although the pathogenesis underlying colonic melanocytic nevi remains unclear, we speculate that colonic melanocytic nevi develop via abnormal migration or differentiation of neural crest cells during embryogenesis.

(c) 2019 The Author(s)

Published by S. Karger AG, Basel
\end{abstract}

\section{Introduction}

Typical melanocytic nevi usually occur in photo-exposed areas, where the risk of cutaneous melanoma greatly increases; these lesions are generally round with a uniform color and a diameter of $\leq 5 \mathrm{~mm}$ [1]. No cases of melanocytic nevi in the colonic mucosa have been reported. However, a few review articles have suggested that colonic melanocytic nevi have the potential to transform into malignant melanoma, like melanocytic nevi of the skin [2]. Melanocytic 
nevus of the colonic mucosa may therefore have potential clinical significance. Here, we report the first detailed case of a colonic melanocytic nevus. The possibility of malignant transformation and potential pathogenesis underlying colonic melanocytic nevi are discussed.

\section{Case Report}

A 54-year-old woman with an unremarkable medical history visited our hospital for screening colonoscopy, which showed a pigmented flat area occupying a quarter of the colonic wall in the ascending colon (Fig. 1a, b). Biopsy of the pigmented lesion was performed. No other abnormalities were found extending into the upper alimentary tract to the duodenum by endoscopy.

Histopathological examination of the biopsy specimen revealed focal proliferation of pigmented cells in small nests in the lamina propria of the colonic mucosa. These pigmented cells showed scant nuclear atypia, and no mitotic figures were observed (Fig. 1c, d). Immunohistochemistry was performed using an auto-immunostaining system (Dako EnVision System, Denmark) for S-100 protein (polyclonal, Dako, Denmark), Melan-A (M2-7C10+M2-9E3, DBS, CA, USA), and Ki-67 (MIB-1, Dako, Denmark). Immunohistochemical staining revealed that many of the pigmented cells were positive for S-100 and Melan-A expression (Fig. 1e). The Ki67 labeling index was very low, and hematoxylin-eosin staining confirmed that the cells were melanocytes (Fig. 1f). Therefore, the final diagnosis was melanocytic nevus of the colon, with no malignant cells.

\section{Discussion}

Melanocytic nevus of the colonic mucosa has not been previously reported in the literature; this is the first reported case. In contrast, a few reports of colonic malignant melanoma have been published, and it has been hypothesized that melanocytic nevi are potential precursor lesions of malignant melanoma, as well as cutaneous lesions [2]. However, we considered the probability of development of colonic malignant melanoma from colonic melanocytic nevus to be low, because melanocytic nevus of the colon is extremely rare, and no cases of colonic malignant melanoma accompanied by a melanocytic nevus surrounding the mucosa have been reported [3].

Although the pathogenesis of colonic melanocytic nevus remains unclear, it may involve abnormalities in the migration and differentiation of trunk neural crest cells. Migration of trunk neural crest cells may occur via a dorsolateral pathway and/or a ventral pathway. Neural crest cells migrating through the dorsolateral pathway may disseminate into the epidermis during embryogenesis, where they differentiate into melanocytes. In contrast, neural cells migrating via the ventral pathway may disperse into the alimentary tract, where they differentiate into neural cells [4]. Two hypotheses regarding the pathogenesis of melanocytic nevus of the colon via abnormal migration and differentiation of neural crest cells are proposed below $[2,5]$.

The first hypothesis involves the abnormal migration of neural crest cells. In general, melanocytes that develop from neural crest cells migrate through the dorsolateral pathway. The migration of neural crest cells might be regulated by interactions between several proteins, such as ephrin, fibronectin, laminin, and integrin [6, 7]. In particular, expression of ephrin-B1, a subtype of ephrin, may promote expression of integrin and induce the migration of neural 
crest cells via the dorsolateral pathway [7]. Thus, aberrant expression of these proteins might lead to migration of melanocytes into the alimentary tract.

The second hypothesis involves the abnormal differentiation of neural crest cells. Several genes have been suggested to be associated with neural crest cell differentiation. For example, expression of Sox10 may activate several cell-type-specific differentiation genes in melanogenic neural crest cells, including Mitf, c-kit, and Cx32 [8]. Aberrant expression of Sox10 might cause differentiation of neural crest cells to melanocytes in the alimentary tract. As a result, melanocytes might disseminate into the intestine during embryogenesis. Accordingly, we speculate that colonic melanocytic nevi might develop from abnormal expression of unidentified genes involved in the differentiation of neural crest cells.

In conclusion, to the best of our knowledge, we experienced the first case of colonic melanocytic nevus. Although potential mechanisms underlying the pathogenesis of colonic melanocytic nevi remain unclear, we have proposed two hypotheses involving abnormal migration and differentiation of neural crest cells.

\section{Statement of Ethics}

We have not submitted an ethical institutional review board statement.

\section{Disclosure Statement}

The authors declare no conflicts of interest.

\section{Funding Sources}

The authors declare that there are no funding sources.

\section{Author Contributions}

Noriyuki Uesugi, Ryo Sugimoto, and Tamotsu Sugai designed the study; Hiroo Tateno, Hiroshi Yokoyama, and Shu Abe collected clinical data; and Noriyuki Uesugi and Tamotsu Sugai analyzed the data and wrote the manuscript.

\section{References}

1 Tokuda Y, Saida T, Murata H, Murase S, Oohara K. Histogenesis of congenital and acquired melanocytic nevi based on histological study of lesion size and thickness. J Dermatol. 2010 Dec;37(12):1011-8.

2 Khalid U, Saleem T, Imam AM, Khan MR. Pathogenesis, diagnosis and management of primary melanoma of the colon. World J Surg Oncol. 2011 Feb;9(1):14.

3 Blecker D, Abraham S, Furth EE, Kochman ML. Melanoma in the gastrointestinal tract. Am J Gastroenterol. 1999 Dec;94(12):3427-33.

4 Okun MR. Histogenesis of melanocytes. J Invest Dermatol. 1965 May;44(5):285-99.

5 Jacobs-Cohen RJ, Wade PR, Gershon MD. Suppression of the melanogenic potential of migrating neural crestderived cells by the branchial arches. Anat Rec. 2002 Sep;268(1):16-26.

6 Lallier T, Bronner-Fraser M. Inhibition of neural crest cell attachment by integrin antisense oligonucleotides. Science. 1993 Jan;259(5095):692-5. 


\section{Case Reports in Gastroenterology}

\begin{tabular}{l|l}
\hline Case Rep Gastroenterol 2019;13:271-274 \\
\hline DOI: 10.1159/000501196 & $\begin{array}{l}\text { @ 2019 The Author(s). Published by S. Karger AG, Basel } \\
\text { www.karger.com/crg }\end{array}$ \\
\hline
\end{tabular}

Uesugi et al.: Colonic melanocytic nevus

7 Santiago A, Erickson CA. Ephrin-B ligands play a dual role in the control of neural crest cell migration. Development. 2002 Aug;129(15):3621-32.

8 Meulemans D, Bronner-Fraser M. Gene-regulatory interactions in neural crest evolution and development. Dev Cell. 2004 Sep;7(3):291-9.
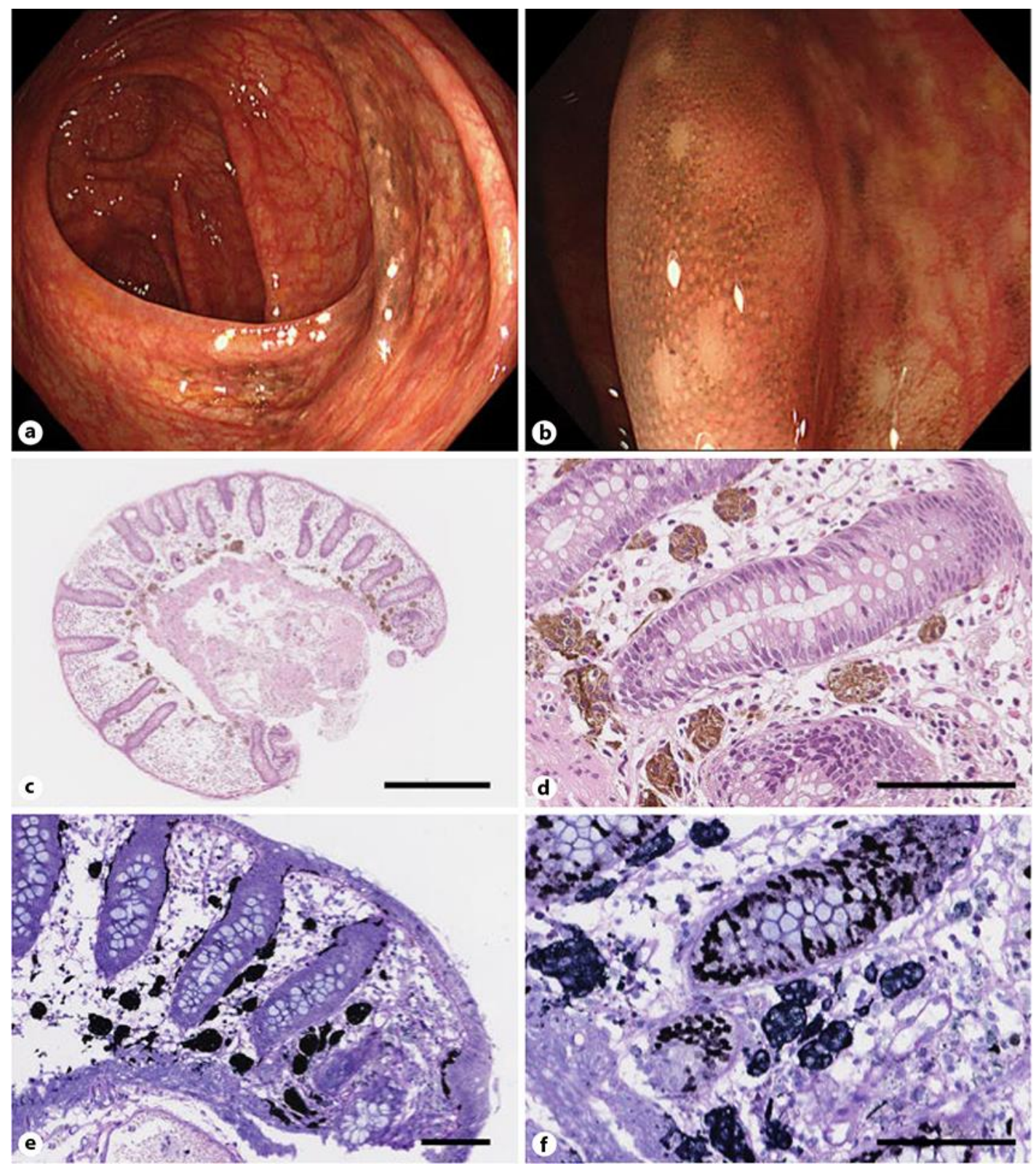

Fig. 1. a, b Endoscopic finding of the ascending colon. A pigmented flat lesion is visible at the contralateral side of Bauhin's valve. $\mathbf{c}-\mathbf{f}$ Histological findings of an endoscopically acquired biopsy specimen. Proliferation of many agglomerated melanocytes with brown pigmentation was observed in the lamina propria of the colonic mucosa. Scale bar: $500 \mu \mathrm{m}$ (c) and $100 \mu \mathrm{m}$ (d). Melanocytes were positive for Melan-A by immunohistochemistry and the Ki-67 labeling index was very low. Scale bar: $100 \mu \mathrm{m}(\mathbf{e}, \mathbf{f})$, 